\title{
Selected Topics in Homogenization of Transport Processes in Historical Masonry Structures
}

\author{
Jan Sýkora ${ }^{\mathrm{a}}$, Jan Zeman ${ }^{\mathrm{a}, \mathrm{b}}$, and Michal Šejnoha ${ }^{\mathrm{a}, *}$ \\ ${ }^{a}$ Department of Mechanics, Faculty of Civil Engineering, Czech Technical University in Prague, Thákurova 7, 16629 \\ Prague 6, Czech Republic \\ ${ }^{b}$ Centre of Excellence IT4Innovations, VŠB-TU Ostrava, 17. listopadu 15/2172 70833 Ostrava-Poruba, Czech Republic
}

\begin{abstract}
The paper reviews several topics associated with the homogenization of transport processed in historical masonry structures. Since these often experience an irregular or random pattern, we open the subject by summarizing essential steps in the formulation of a suitable computational model in the form of Statistically Equivalent Periodic Unit Cell (SEPUC). Accepting SEPUC as a reliable representative volume element is supported by application of the Fast Fourier Transform to both the SEPUC and large binary sample of real masonry in search for effective thermal conductivities limited here to a steady state heat conduction problem. Fully coupled non-stationary heat and moisture transport is addressed next in the framework of two-scale first-order homogenization approach with emphases on the application of boundary and initial conditions on the meso-scale.
\end{abstract}

Keywords: Random masonry, Binary images, Statistically equivalent periodic unit cell, Computational homogenization, Fast Fourier Transform, Coupled heat and moisture transport.

\section{INTRODUCTION}

Advanced computational simulations of historic structures are becoming increasingly common in the assessment of their existing state and in planning of reconstruction measures [1]. In this context, particular attention needs to be paid to variations of temperature and moisture fields, whose contribution to structural damage usually far exceeds the effects of mechanical loadings, see e.g. [2-4] for concrete case studies. Taking into account highly heterogeneous character of historical constructions, simulations of these phenomena often necessitates the deployment of multi-scale strategies, developed for mechanical [5- 8] and transport processes $[9,10]$ in masonry structures. Successful engineering application of this modeling approach to the assessment of Charles Bridge in Prague is described in our work [11]. There, three-dimensional multi-physics analysis of the bridge body was executed, with material parameters at the macroscopic structural scale determined from meso-scale simulations. Among other things, results of the study highlighted the need for fully coupled macro-meso simulations of transport processes. In this paper, we complement these results by a more detailed analysis of two aspects of multiscale simulations, namely the meso-scale representation for irregular masonry structures and the introduction of proper boundary and initial conditions in the macro-meso scale transition.

*Address correspondence to this author at the CTU in Prague, Faculty of Civil Engineering, Department of mechanics, Thákurova 7, 16629, Praha 6, Czech Republic; Tel: +420224354494; Fax: +420224310775;

E-mail: sejnom@fsv.cvut.cz
The masonry texture at meso-scale is to be incorporated in the form of the Representative Volume Element (RVE), a statistically representative sample of the analyzed material [12]. Specification of the RVE is particularly simple in case of a regular masonry, for which it reduces to a Periodic Unit Cell (PUC) associated with a given type of the bond [5]. However, historical masonry structures are typically of irregular or random textures, which renders the determination of PUC unambiguous. A convenient approach to overcome this difficulty was introduced by Povirk [13], who suggested to replace the original complex meso-structure with an idealized PUC, with parameters determined by matching spatial statistics of original and simplified representation. By combining his ideas with related works on microstructure reconstruction, e.g. [14], the concept of Statistically Equivalent Periodic Unit cell (SEPUC) was later successfully applied to, e.g. analysis of fiber-reinforced $[15,16]$ and woven composites [17, 18], high-density polydisperse particle packings [19] or micro-heterogeneous steels [20]. In Section 2, we employ this procedure to an irregular masonry wall of Charles Bridge in Prague, characterized by a digital photograph.

Generating a suitable RVE of a heterogeneous material is just the first step towards a reliable prediction of material as well as structural response of masonry. Focusing on the description of transport processes in heterogeneous media the reader is advised to study the work by Özdemir et al. [21] and Larsson et al. [22]. Through the application of consistent variational formulation the authors in [22] suggested the macroscopic response to be dependent on the actual size of mesoscopic RVE, providing the transient conditions are assumed on both the macro- and meso-scale. Being aware of 
the need for a fully coupled multi-scale analysis of simultaneous heat and moisture transport in masonry structures performed recently in [23], it becomes clear that additional sub-stepping of a given macroscopic time step on the mesoscale may considerably slow down the computational process. To show that in some cases this step might be avoided by running the meso-scale analysis under steady state conditions [21] even for a finite size RVE may thus prove useful in keeping the computational cost relatively low. This issue is addressed in the second part of this paper, Section 3, with particular attention dedicated to the influence of loading and initial conditions imposed on the meso-scale.

\section{IMAGE-BASED GEOMETRICAL MODELING}

This section deals with the first aspect of multi-scale simulations announced above, namely with the realistic representation of irregular masonry structure relying on imagebased data. To this purpose, in Section 2.1 we introduce the model of an idealized mesostructure and the procedure to determine its parameters. Section 2.2 briefly reviews the numerical scheme employed to determine the local fields operating directly on mesostructural images. These two tools are combined together in Section 2.3 to assess the SEPUC quality in view of the distribution of heat fluxes in disordered masonry under steady-state conditions.

\subsection{Strategy of SEPUC Determination}

The key step in the SEPUC definition is a proper choice of spatial statistics to characterize the dominant features of the heterogeneous material under study. With the focus on masonry structures, we limit our attention to the mortar twopoint probability function, see e.g. [24] for a general overview and examples of alternative statistical descriptors.

To introduce the subject, consider a masonry sample $\Omega$ composed of mortar and blocks, and denote the characteristic function of the domain occupied by mortar as $\chi(\boldsymbol{x})$. Then, the two-point probability function $S_{2}$ states the probability that two points $\boldsymbol{x}$ and $\boldsymbol{y}$, randomly thrown into a medium, will both be found in the mortar phase,

$S_{2}(\boldsymbol{x}, \boldsymbol{y})=\mathbb{P}(\chi(\boldsymbol{x}) \chi(\boldsymbol{y})=1)$.

For the case of statistically homogeneous and ergodic media, two-point probability function depends on $(x-y)$ only and $S_{2}(0)=\phi$, where $\phi$ is the mortar volume fraction. Moreover, it can be obtained from the relation

$S_{2}=\frac{1}{|\Omega|} \mathcal{F}^{-1}(\mathcal{F}(\chi) \overline{\mathcal{F}(\chi)})$,

that can be efficiently evaluated using the Fast Fourier Transform (FFT) techniques for image-based microstructures [24, 25]. In Eq. (2), $\mathcal{F}(\bullet)$ and $\mathcal{F}^{-1}(\bullet)$ designate the forward and inverse Fourier Transform operators, respectively, - denotes the complex conjugate and $|\Omega|$ is the area of $\Omega$.

Once the original structure has been quantified by a suitable statistical descriptor, a proper parametrization of the idealized cell geometry needs to be introduced, expressed here by a parameter vector $\boldsymbol{p}$. Its optimal value then follows from the minimization of a least square error
$E(\boldsymbol{p})=\frac{1}{\left|\Omega_{\square}\right|} \int_{\Omega_{\square}}\left(\overline{S_{2}}(\boldsymbol{x})-S_{2}(\boldsymbol{x}, \boldsymbol{p})\right)^{2} \mathrm{~d} x$,

expressed as the difference between the target statistical descriptor $\bar{S}_{2}(\boldsymbol{x})$ related to the original microstructure and the unit cell associated with $\boldsymbol{p}$, integrated over the unit cell domain $\Omega_{\square} \subset \Omega$.

A closer inspection reveals that the objective function (3) is non-convex, multimodal and discontinuous due to the effect of limited bitmap resolution. Based on our previous experience [26, 27], a global stochastic optimization algorithm, relying on the combination of real-valued genetic algorithms and the Simulated Annealing method, is employed to solve this optimization problem.

\subsection{Homogenization Scheme}

The distribution of locafields within $\Omega_{\square}$ follows from the solution of a periodic unit cell problem [28]

$\boldsymbol{\nabla} \times \boldsymbol{\nabla} \theta=0, \boldsymbol{\nabla} \cdot \boldsymbol{q}=0, \boldsymbol{q}=-\lambda \boldsymbol{\nabla} \theta$ in $\Omega_{\square}$,

in which $\boldsymbol{q}$ stands for the thermal flux vector, $\lambda$ is the secondorder tensor of material conductivity and $\boldsymbol{\nabla} \theta$ denotes the $\Omega_{\square}$ - periodic field of temperature gradient satisfying

$\frac{1}{\left|\Omega_{\square}\right|} \int_{\Omega_{\square}} \nabla \theta(x) \mathrm{d} \boldsymbol{x}=\boldsymbol{\nabla} \Theta$,

where $\boldsymbol{\nabla} \Theta$ is the macroscopic temperature gradient prescribed over $\Omega_{\square}$, see Section 3 below for additional details. It is well-known that the solution to the unit cell satisfies the Lippman-Schwinger equation [28]

$\boldsymbol{\nabla} \theta(\boldsymbol{x})+\int_{\Omega_{\square}} \boldsymbol{\Gamma}(\boldsymbol{x}-\boldsymbol{y}) \delta \boldsymbol{\lambda}(\boldsymbol{y}) \boldsymbol{\nabla} \theta(\boldsymbol{y}) \mathrm{d} \boldsymbol{y}=\boldsymbol{\nabla} \Theta$,

where $\delta \boldsymbol{\lambda}=\boldsymbol{\lambda}-\lambda^{(0)} \boldsymbol{I}, \lambda_{0}$ is the conductivity of an auxiliary isotropic reference medium and the second-order operator $\boldsymbol{\Gamma}$ is related to the Green function of problem (4) with $\lambda(\boldsymbol{x})=$ $\lambda^{(0)} \boldsymbol{I}$. It admits a compact closed-form expression in the Fourier space [28]

$\mathcal{F}(\Gamma)(\boldsymbol{k})=\left\{\begin{array}{c}\mathbf{0} \text { for } \boldsymbol{k}=\mathbf{0}, \\ \frac{\boldsymbol{k} \otimes \boldsymbol{k}}{\boldsymbol{k} \cdot \boldsymbol{k}} \text { otherwise, }\end{array}\right.$

so that its action can be efficiently evaluated by the FFT algorithm. This observation resulted in an iterative scheme due to Moulinec and Suquet [29], applicable to arbitrary digitized media.

In our case, we adopt an accelerated version of the original algorithm due to Zeman et al. [30]. Since the sample is discretized by a regular $N_{1} \times N_{2}$ bitmap, it is convenient to project the integral equation (6) onto the space of trigonometric polynomials, e.g. [31]. This yields the linear system in the form

$(\boldsymbol{I}+\boldsymbol{B}) \nabla \boldsymbol{\theta}_{\mathrm{d}}=\nabla \boldsymbol{\Theta}_{\mathrm{d}}$,

where the $2 N_{1} N_{2}$ vector $\nabla \boldsymbol{\theta}_{\mathrm{d}}$ stores the unknown discrete values of temperature gradient at pixels, $\nabla \boldsymbol{\Theta}_{\mathrm{d}}$ is the corresponding vector of the overall temperature gradient and the matrix $\boldsymbol{B}$ is expressed as

$\boldsymbol{B}=\left(\begin{array}{cc}\boldsymbol{F}^{-1} & \mathbf{0} \\ \mathbf{0} & \boldsymbol{F}^{-1}\end{array}\right)\left(\begin{array}{ll}\boldsymbol{\Gamma}_{11} & \boldsymbol{\Gamma}_{12} \\ \boldsymbol{\Gamma}_{21} & \boldsymbol{\Gamma}_{22}\end{array}\right)\left(\begin{array}{ll}\boldsymbol{F} & \mathbf{0} \\ \mathbf{0} & \boldsymbol{F}\end{array}\right)\left(\begin{array}{ll}\delta \boldsymbol{\lambda}_{11} & \delta \boldsymbol{\lambda}_{12} \\ \delta \boldsymbol{\lambda}_{21} & \delta \boldsymbol{\lambda}_{22}\end{array}\right)$. 


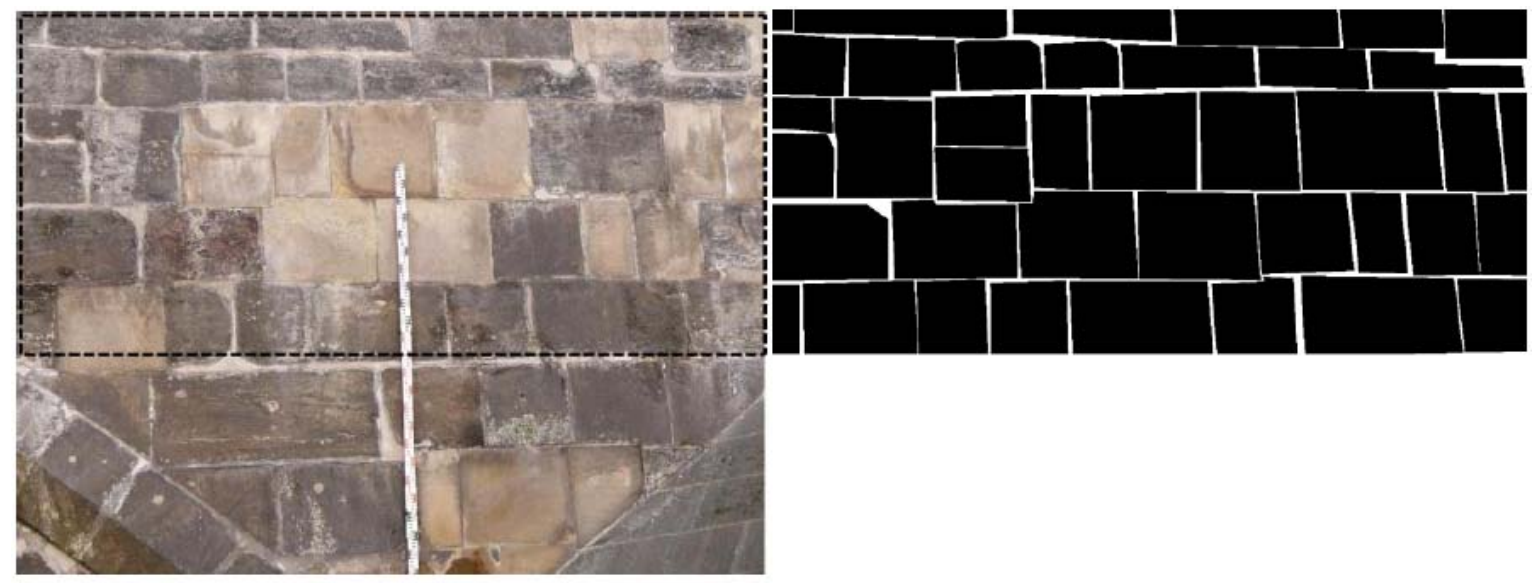

(a)

Fig. (1). (a) Example of a masonry wall of Charles Bridge in Prague and (b) meso-structural window $\Omega$ provided in the form of $1,600 \times 735$ black-and-wide bitmap.

Here, the matrices $\boldsymbol{F}$ and $\boldsymbol{F}^{-1}$ implement the forward and inverse discrete Fourier transform and, e.g. $\delta \boldsymbol{\lambda}_{12}$ is a diagonal $\left(N_{1} N_{2}\right) \times\left(N_{1} N_{2}\right)$ matrix storing the corresponding component of the conductivity matrix at individual pixels, see [30] for more details. The system (8) is solved using standard conjugate gradient algorithm.

Upon convergence, the distribution of the local heat flux $\boldsymbol{q}$ due to $\boldsymbol{\nabla} \Theta$ is determined from the solution $\boldsymbol{\nabla} \theta$ by Eq. (4) ${ }_{3}$ and the global heat flux is computed as

$\boldsymbol{Q}=\frac{1}{\left|\Omega_{\square}\right|} \int_{\Omega_{\square}} \boldsymbol{q}(\boldsymbol{x}) \mathrm{d} \boldsymbol{x}$.

This allows us to determine the $2 \times 2$ homogenized conductivity matrix $\lambda^{\text {hom }}$ via the solution of two successive steady state heat conduction problems. To that end, the periodic unit cell is loaded, in turn, by each of the two components of $\nabla \Theta$ equal to unity, while the remaining one vanishes. The corresponding volume flux averages $\boldsymbol{Q}$ then provide individual columns of $\lambda^{\text {hom }}$.

\subsection{Example}

Principles of the introduced methodology are illustrated by the analysis of sandstone facing masonry wall of Charles Bridge in Prague appearing in Fig. (1). As the first step, the original color image, Fig. (1a), was thresholded to the binary representation, manually adjusted to remove image processing artifacts and a rectangular domain $\Omega$ was selected for further analysis, Fig. (1b).

Our aim is to replace this complex meso-structure with its idealized representation in terms of a two-block-layer SEPUC, described by twelve parameters assembled in the vector

$\boldsymbol{p}=\left(b, b_{1}, b_{2}, \Delta, h_{1}, h_{2}, t_{1}, t_{2}, t_{3}, t_{4}, t_{5}, t_{6}\right)$,

see Fig. (2a). These parameters are adjusted to minimize the discrepancy between the target two-point probability function, Fig. (3a), and the one corresponding to the SEPUC, Fig. (3b). As visible, the resulting SEPUC-based representation $\Omega_{\square}$, Fig. (2b), captures the dominant features of the original microstructures, such as the overall volume fraction equal to $7.5 \%$, or the average thickness of joints, corresponding to the spread of the peak at $\boldsymbol{x}=\mathbf{0}$ in the horizontal and vertical directions. However, a significant amount of finescale features of the two-point probability functions, visible in the original data, are filtered out or poorly reproduced by the adopted, more regular, representation.

Of course, the impact of such approximation needs to be quantified a-posteriori, from the point of view of the particular application. In our case, such a step is performed on the basis of homogenized conductivities and distribution of heat fluxes as determined by the FFT scheme. The distribution of the magnitudes of local fields due to macroscopic temperature gradient $\partial \Theta / \partial x_{2}$ appears in Fig. (4), for phase conductivities set according to Table 2 . We observe that even though the local fields within the SEPUC are certainly more regular than in the original mesostructure, the extreme values as well as the average distributions are reproduced surprisingly well.

This claim is further supported by very close match between the homogenized conductivities of SEPUC and the original media, Table 1, demonstrating that the SEPUC is capable of reproducing almost perfect isotropy of the original sample. Finally, for the sake of comparison, we also present homogenized conductivities of unit cells used in the following section, cf. Fig. (5). The influence of the degree of heterogeneity on the resulting predictions is evident.

\section{MULTI-SCALE HOMOGENIZATION OF COU- PLED HEAT AND MOISTURE TRANSPORT}

In this section, we continue with the selected example of Charles Bridge and extend the previous study to the multiscale modeling of coupled nonlinear transient heat and moisture transport in masonry structures. While still adopting the first order homogenization approach (linear variation of macroscopic temperature and moisture fields is assumed) we choose, unlike the previous section, the finite element method (FEM) to solve the resulting system of partial differential equations. In the present study, these arise from the application of a nonlinear diffusion model proposed by Künzel in $[32,33]$.The model is described by the energy balance equation 
Table 1. Homogenized Thermal Conductivities in $\left[\mathrm{Wm}^{-1} \mathrm{~K}^{-1}\right]$

\begin{tabular}{|l|r|r|r|}
\hline & $\lambda_{\mathbf{2 2}}^{\text {hom }}$ & $\lambda_{\mathbf{1 2}}^{\text {hom }}=\lambda_{\mathbf{2 1}}^{\text {hom }}$ & \multicolumn{1}{|c|}{$\lambda_{\mathbf{2 2}}^{\text {hom }}$} \\
\hline \hline Original mesostructure & 0.2612 & 0.0000 & 0.2622 \\
SEPUC & 0.2616 & 0.0000 & 0.2618 \\
\hline Regular masonry & 0.2856 & 0.0000 & 0.2967 \\
Irregular masonry & 0.2932 & -0.0015 & 0.2960 \\
\hline
\end{tabular}

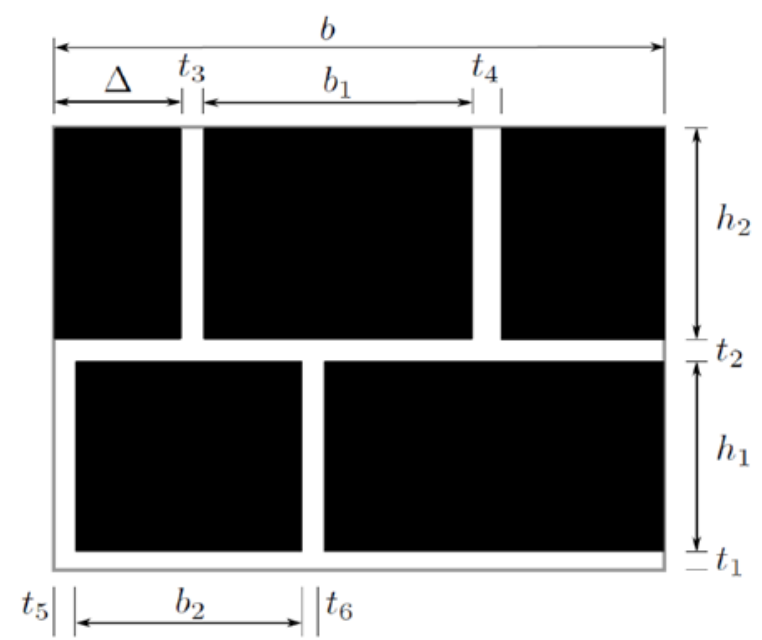

(a)

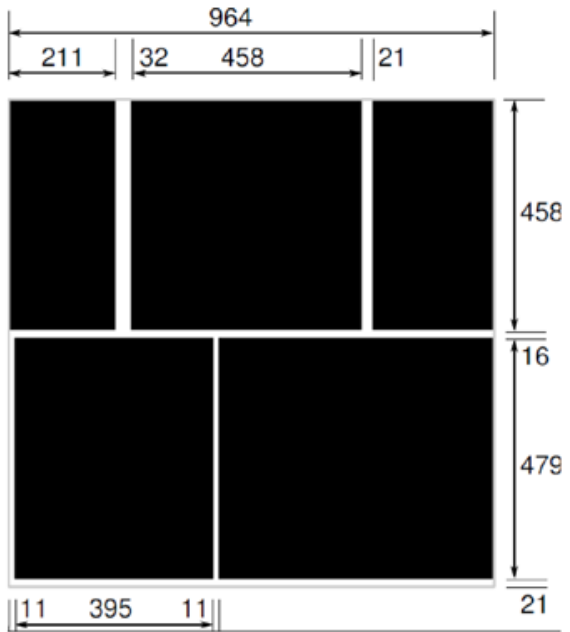

(b)

Fig. (2). Determination of SEPUC for irregular masonry; (a) adopted parametrization and (b) optimal mesostructure with dimensions in $[\mathrm{mm}]$.

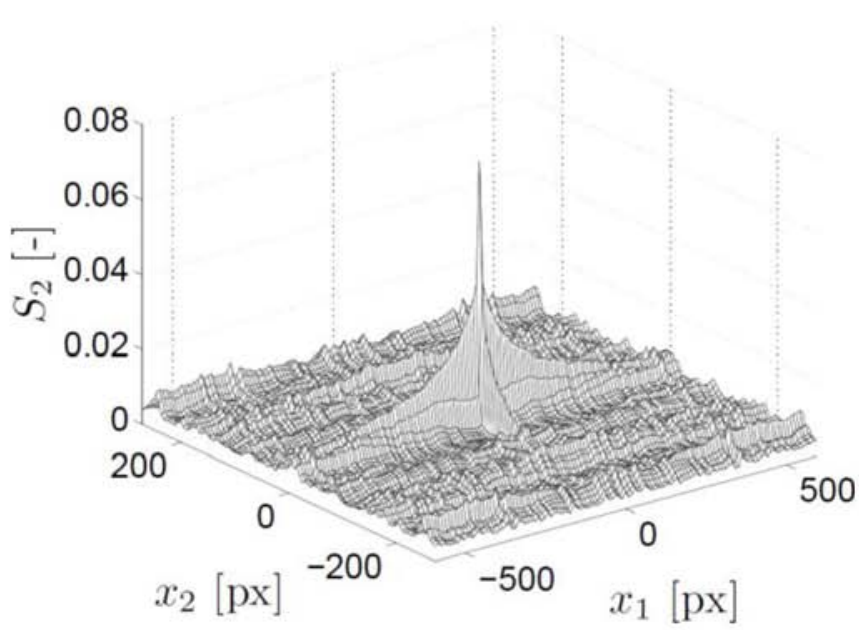

(a)

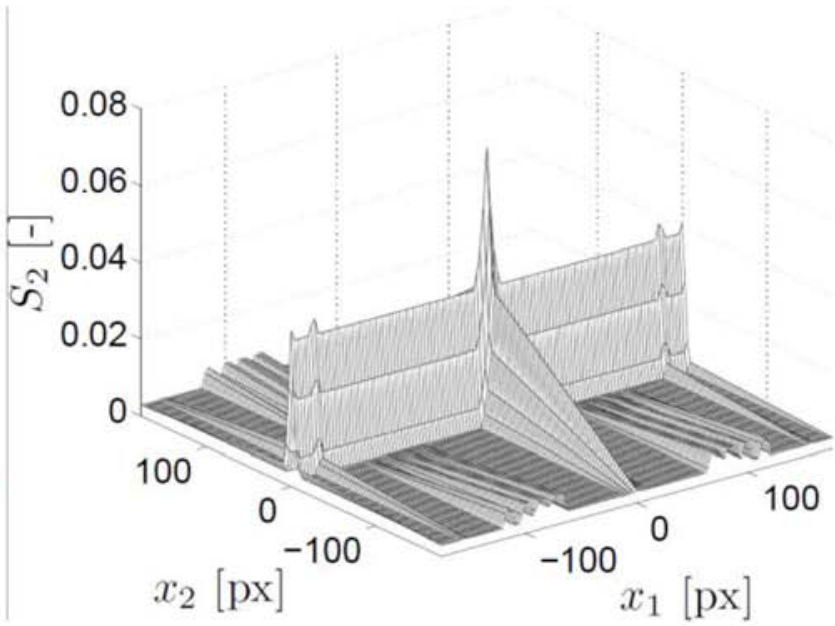

(b)

Fig. (3). Two-point probability function describing (a) target microstructure and (b) SEPUC (1 pixel corresponds to $\sim 2.6 \mathrm{~mm}$ ).

$\frac{\mathrm{d} H}{\mathrm{~d} \theta} \frac{\mathrm{d} \theta}{\mathrm{d} t}=\boldsymbol{\nabla}^{\mathrm{T}}[\lambda \boldsymbol{\nabla} \theta]+h_{v} \boldsymbol{\nabla}^{\mathrm{T}}\left[\delta_{p} \boldsymbol{\nabla}\left\{\varphi p_{\text {sat }}(\theta)\right\}\right]$,

and by the mass conservation equation

$\frac{\mathrm{d} w}{\mathrm{~d} \varphi} \frac{\mathrm{d} \varphi}{\mathrm{d} t}=\nabla^{\mathrm{T}}\left[D_{\varphi} \boldsymbol{\nabla} \varphi\right]+\nabla^{\mathrm{T}}\left[\delta_{p} \boldsymbol{\nabla}\left\{\varphi p_{\mathrm{sat}}(\theta)\right\}\right]$

where temperature $\theta$ and relative humidity $\varphi$ are the two state variables. The model requires specifying the enthalpy of the moist building material $H$, the water content of the building material $w$, the coefficient of thermal conductivity $\lambda$, the liquid conduction coefficient $D_{\varphi}$, the water vapor permeability $\delta_{p}$, the evaporation enthalpy of water $h_{v}$ and the water vapor saturation pressure $p_{\text {sat }}$. Details regarding the functional dependence of the model parameters on temperature and relative humidity are available, e.g. in [32, 34].

In [34] the authors noticed a significant dependence of the homogenized macroscopic properties on the applied temperature and relative humidity gradients. This finding promoted the solution of full scale analysis of masonry struc- 
tures in a fully coupled multi-scale homogenization framework. To that end, a nested $\mathrm{FE}^{2}$ scheme, graphically presented in Fig. (5) (see also [21, 22, 23] for more details), appears as a suitable method of attack. In such a case we expect the homogenized macro-scale fields to be found from the solution of a certain sub-scale (meso-scale) problem performed on an RVE loaded by the prescribed constant temperatures and moisture gradients.

As already mentioned in the introductory part, Larson et al. [22] have shown that assuming a non-stationary response also on meso-scale generates a non-local term in the homog- enized macroscopic equations, which renders the macroscopic response dependent on the RVE size. There is no dispute that performing the non-stationary analysis on both scales may considerably increase the computational cost making the numerical analysis prohibitively expensive even if exploiting parallelization. To reconcile these issues for a typical masonry material and RVE sizes is thus crucial for the success of analysis of full scale tree-dimensional models of historical masonry structures such as Charles Bridge. This will be the main topic of subsequent paragraphs addressing also the effect of loading, boundary and initial conditions.

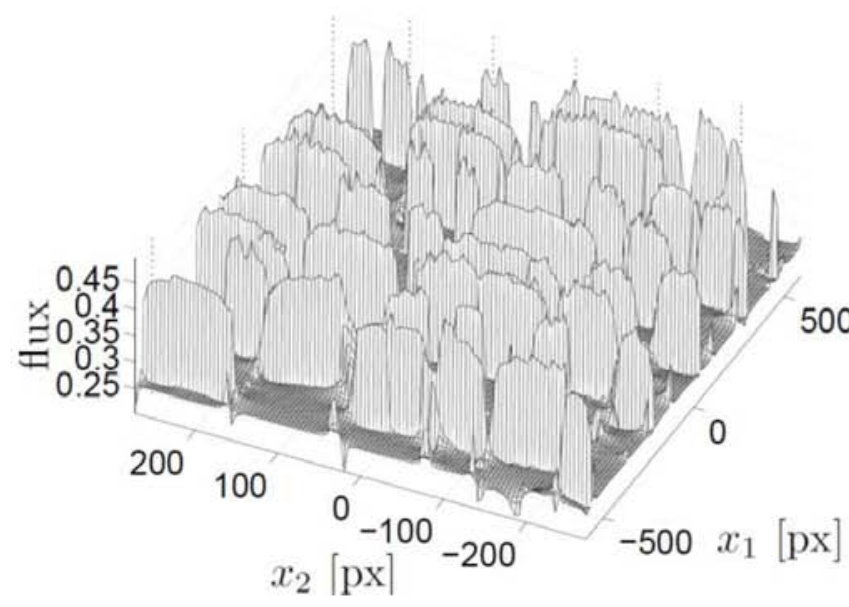

(a)

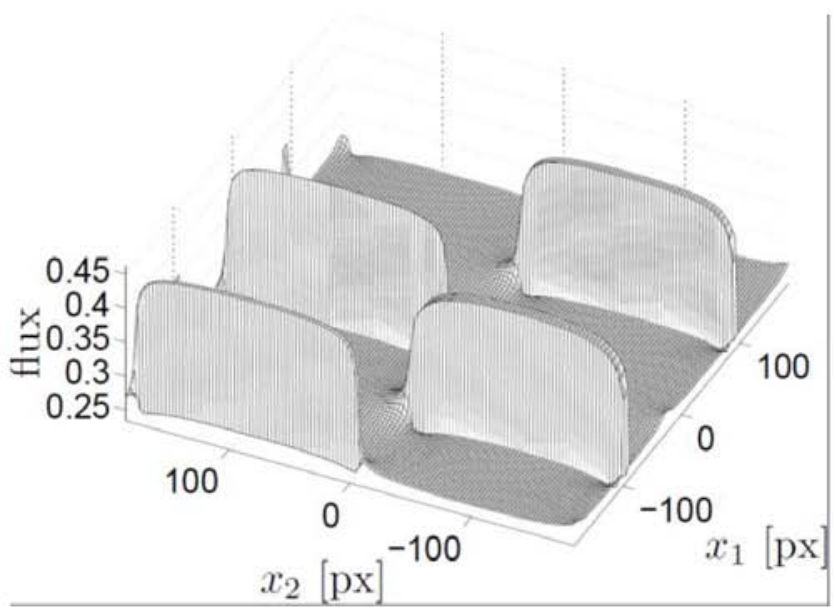

(b)

Fig. (4). Distribution of heatflux magnitudes $\|\mathrm{q}\|$ (in $\left[\mathrm{Wm}^{-1}\right]$ ) due to due to macroscopic temperature gradient $\partial \Theta / \partial x_{2}=1\left[\mathrm{Km}^{-1}\right]$ in (a) original domain and (b) SEPUC.

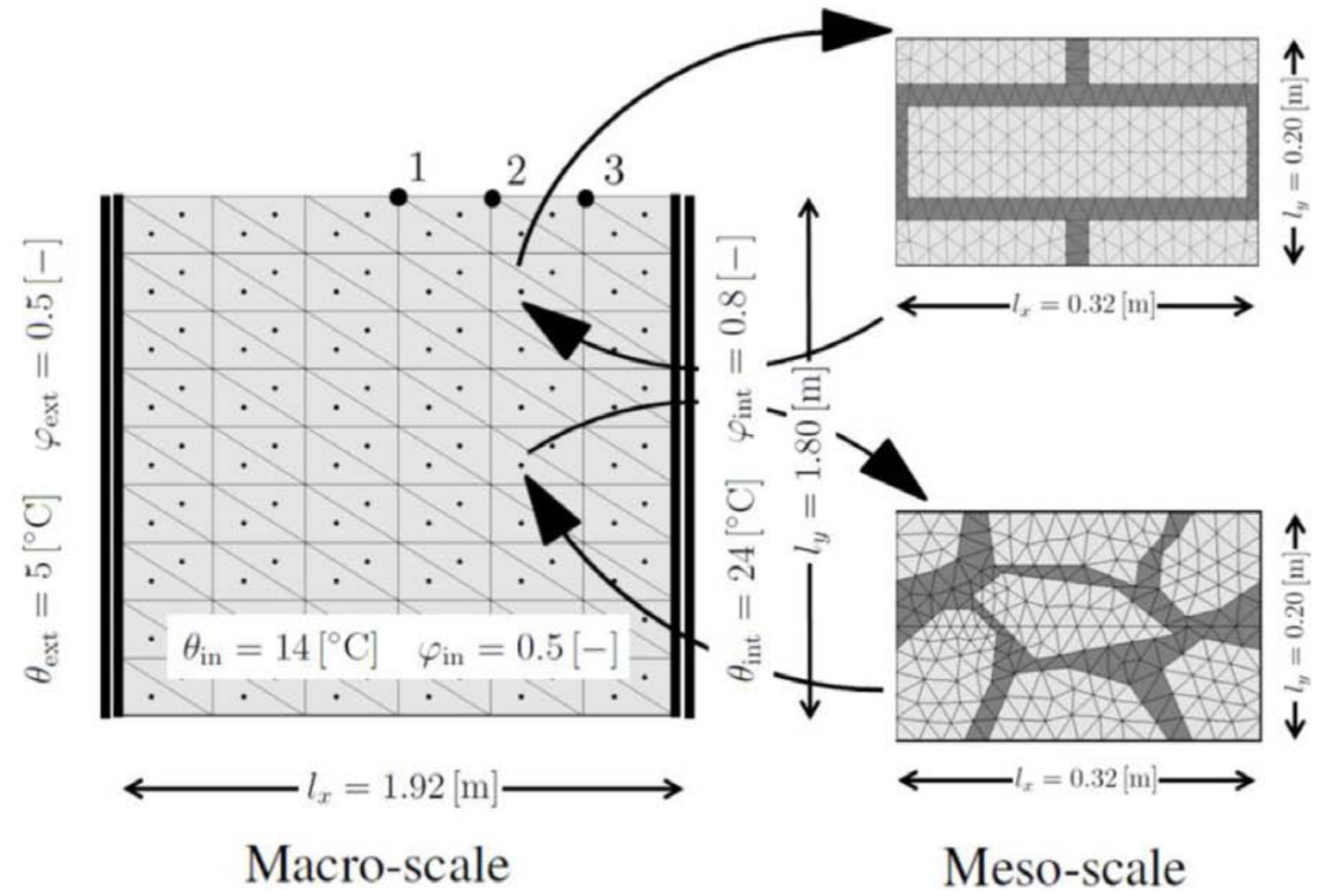

Fig. (5). Scheme of coupled multi-scale framework. 
Since irrelevant from the computational point of view we consider, henceforth, only the regular (PUC) and irregular (SEPUC) bonding of masonry displayed in Fig. (5).

\subsection{Theoretical Formulation}

To begin, we adopt a variationally consistent homogenization outlined in detail in [22] starting with the assumption that a local field can be replaced by a spatially homogenized one $\langle a\rangle$ such that

$$
\begin{aligned}
& \int_{\Omega} a \mathrm{~d} \Omega \approx \int_{\Omega}\langle a\rangle_{\square} \mathrm{d} \Omega=\int_{\Omega}\left(\frac{1}{\left|\Omega_{\square}\right|} \int_{\Omega_{\square}} a \mathrm{~d} \Omega_{\square}\right) \mathrm{d} \Omega, \\
& \int_{\Gamma} a \mathrm{~d} \Gamma \approx \int_{\Omega}\langle a\rangle_{\square} \mathrm{d} \Gamma=\int_{\Gamma}\left(\frac{1}{\left|\Gamma_{\square}\right|} \int_{\Gamma_{\square}} a \mathrm{~d} \Gamma_{\square}\right) \mathrm{d} \Gamma,
\end{aligned}
$$

where $\Omega_{\square}$ and $\Gamma_{\square}$ represent the internal and boundary parts of PUC. In what follows, for the sake of lucidity, we shall treat only the energy balance equation (12) which upon employing Eqs. (14) and (15) becomes

$$
\begin{aligned}
& \int_{\Omega}\left\langle\delta \theta \frac{\mathrm{d} H}{\mathrm{~d} \theta} \frac{\mathrm{d} \theta}{\mathrm{d} t}\right\rangle_{\square} \mathrm{d} \Omega+ \\
& \int_{\Omega}\left\langle\nabla \delta \theta^{\mathrm{T}}\left[\lambda \nabla \theta+h_{v}\left\{\delta_{p} \varphi \frac{\mathrm{d} p_{\mathrm{sat}}}{\mathrm{d} \theta} \nabla \theta\right\}\right]\right\rangle_{\square} \mathrm{d} \Omega+ \\
& +\int_{\Omega}\left\langle\nabla \delta \theta^{\mathrm{T}}\left[h_{v}\left\{\delta_{p} p_{\text {sat }} \nabla \varphi\right\}\right]\right\rangle_{\square} \mathrm{d} \Omega-\int_{\Gamma_{\theta} \bar{q}}\left\langle\delta \theta \bar{q}_{v}\right\rangle_{\square} \mathrm{d} \Gamma=0 .
\end{aligned}
$$

In the spirit of the first order homogenization, it is assumed that the macroscopic temperature and relative humidity vary only linearly over PUC. This can be achieved by loading its boundary by the prescribed temperature $\Theta^{\text {hom }}$ and relative humidity $\Phi^{\text {hom }}$ derived from the uniform macroscopic temperature $\nabla \Theta$ and relative humidity $\nabla \Phi$ gradients. In such a case, the local temperature and relative humidity inside PUC admit the following decomposition

$$
\begin{aligned}
& \theta(\boldsymbol{x})=\Theta\left(\boldsymbol{X}^{0}\right)+\nabla \Theta^{\mathrm{T}}\left\{\boldsymbol{x}-\boldsymbol{X}^{0}\right\}+\theta^{*}(\boldsymbol{x})=\Theta^{\mathrm{hom}}(\boldsymbol{x})+ \\
& \theta^{*}(\boldsymbol{x}), \\
& \varphi(\boldsymbol{x})=\Phi\left(\boldsymbol{X}^{0}\right)+\nabla \Phi^{\mathrm{T}}\left\{\boldsymbol{x}-\boldsymbol{X}^{0}\right\}+\varphi^{*}(\boldsymbol{x})=\Phi^{\mathrm{hom}}(\boldsymbol{x})+ \\
& \varphi^{*}(\boldsymbol{x}),
\end{aligned}
$$

where $\theta^{*}(\boldsymbol{x})$ and $\varphi^{*}(\boldsymbol{x})$ are the fluctuations of local fields superimposed onto linearly varying quantities $\Theta^{\text {hom }}(\boldsymbol{x})$ and $\Phi^{\text {hom }}(\boldsymbol{x})$. The temperature $\Theta\left(\boldsymbol{X}^{0}\right)$ and the moisture $\Phi\left(\boldsymbol{X}^{0}\right)$ at the reference point $\boldsymbol{X}^{0}$ are introduced to link the local fields to their macroscopic counterparts. For convenience, the PUC is typically centered at $\boldsymbol{X}^{0}$. Henceforth, the local fluctuations will be demanded to be periodic, i.e. the same values are enforced on the opposite sides of a rectangular PUC. This ensures the scale transition condition, see e.g. [21], which arises upon averaging the micro-temperature gradient over the volume $|\Omega|$ of PUC

$$
\begin{aligned}
& \langle\boldsymbol{\nabla} \theta(\boldsymbol{x})\rangle_{\square}=\frac{1}{\left|\Omega_{\square}\right|} \int_{\Omega_{\square}} \boldsymbol{\nabla} \theta(\boldsymbol{x}) \mathrm{d} \Omega(\boldsymbol{x})= \\
& \nabla \Theta+\frac{1}{\left|\Omega_{\square}\right|} \int_{\Omega_{\square}} \boldsymbol{\nabla} \theta^{*}(\boldsymbol{x}) \mathrm{d} \Omega(\boldsymbol{x}) .
\end{aligned}
$$

Hence we demand the contribution of fluctuation fields to disappear upon volume averaging

$$
\begin{aligned}
& \left\langle\nabla \theta^{*}(\boldsymbol{x})\right\rangle_{\square}=\frac{1}{\left|\Omega_{\square}\right|} \int_{\Omega_{\square}} \nabla \theta^{*}(\boldsymbol{x}) \mathrm{d} \Omega(\boldsymbol{x})= \\
& \frac{1}{\left|\Omega_{\square}\right|} \int_{\Gamma_{\square}} \theta^{*}(\boldsymbol{x}) \boldsymbol{v}(\boldsymbol{x}) \mathrm{d} \Gamma(\boldsymbol{x}),
\end{aligned}
$$

where $v$ stores the components of the outward unit normal to the boundary of PUC being directed in opposite directions on opposite sides of the PUC.

Next, substituting Eq. (17) into Eq. (16) and collecting the terms corresponding to $\delta \Theta^{\text {hom }}$ and $\delta \theta^{*}$ splits the original problem (12) into the homogenized (macro-scale) problem and local sub-scale (meso-scale) problem. Since details on the derivation of equations driving the solution on individual scales have already been given in our proceeding works [10, 23], we present only the result pertinent to the macro-scale

$$
\begin{aligned}
& -\underbrace{\int_{\Omega}\left\langle\delta \Theta^{\mathrm{T}} \frac{\mathrm{d} H}{\mathrm{~d} \theta} \frac{\mathrm{d}}{\mathrm{d} t}\left(\Theta+\nabla \Theta^{\mathrm{T}}\left\{\boldsymbol{x}-\boldsymbol{X}^{0}\right\}\right)\right\rangle_{\square} \mathrm{d} \Omega-}_{\left(\mathbf{C}_{\theta \theta}+\mathbf{C}_{\theta \theta}^{\prime}\right) \frac{\mathrm{d} \boldsymbol{r}_{\theta}}{\mathrm{d} t}} \\
& -\underbrace{\int_{\Omega}\left\langle\delta \nabla \Theta^{\mathrm{T}}\left\{\boldsymbol{x}-\boldsymbol{X}^{0}\right\} \frac{\mathrm{d} H}{\mathrm{~d} \theta} \frac{\mathrm{d}}{\mathrm{d} t}\left(\Theta+\nabla \Theta^{\mathrm{T}}\left\{\boldsymbol{x}-\boldsymbol{X}^{0}\right\}\right)\right\rangle_{\square} \mathrm{d} \Omega}_{\left(\mathbf{c}_{\theta \theta}^{\prime}+\mathbf{C}_{\theta \theta}^{\prime \prime}\right) \frac{\mathrm{d} \boldsymbol{r}_{\theta}}{\mathrm{d} t}}- \\
& -\underbrace{\int_{\Omega}\left\langle\boldsymbol{\nabla} \delta \Theta^{\mathrm{T}}\left[\lambda \boldsymbol{\nabla} \Theta+h_{v}\left\{\delta_{p} \varphi \frac{\mathrm{d} p_{\mathrm{sat}}}{\mathrm{d} \theta} \nabla \Theta\right\}\right]\right\rangle_{\square} \mathrm{d} \Omega}_{\mathbf{K}_{\theta \theta} \boldsymbol{r}_{\theta}}- \\
& -\underbrace{\int_{\Omega}\left\langle\nabla \delta \Theta^{\mathrm{T}}\left[h_{v}\left\{\delta_{p} p_{\text {sat }} \boldsymbol{\nabla} \Phi\right\}\right]\right\rangle_{\square} \mathrm{d} \Omega}_{\mathbf{K}_{\theta \varphi} \boldsymbol{r}_{\varphi}}+\underbrace{\int_{\Gamma_{\theta}^{\bar{q}}}\left\langle\delta \Theta^{\mathrm{T}} \bar{q}_{v}\right\rangle_{\square} \mathrm{d} \Gamma}_{\boldsymbol{q}_{\mathrm{ext}}}=0 .
\end{aligned}
$$

to identify the solution dependence on the actual size of PUC through the second term in the integral (21).

An analogous approach can be applied also to the moisture transport equation (13) to arrive, after classical finite element discretization, into a discretized system of coupled macroscopic heat and moisture equations

$$
\begin{aligned}
& \mathbf{K}_{\theta \theta} \boldsymbol{r}_{\theta}+\mathbf{K}_{\theta \varphi} \boldsymbol{r}_{\varphi}+\left(\mathbf{C}_{\theta \theta}+2 \mathbf{C}_{\theta \theta}^{\prime}+\mathbf{C}_{\theta \theta}^{\prime \prime}\right) \frac{\mathrm{d} \boldsymbol{r}_{\theta}}{\mathrm{d} t}=\boldsymbol{q}_{\mathrm{ext}}, \\
& \mathbf{K}_{\varphi \theta} \boldsymbol{r}_{\theta}+\mathbf{K}_{\varphi \varphi} \boldsymbol{r}_{\varphi}+\left(\mathbf{C}_{\varphi \varphi}+2 \mathbf{C}_{\varphi \varphi}^{\prime}+\mathbf{C}_{\varphi \varphi}^{\prime \prime}\right) \frac{\mathrm{d} \boldsymbol{r}_{\varphi}}{\mathrm{d} t}=\boldsymbol{g}_{\mathrm{ext}},
\end{aligned}
$$

which have to be properly integrated in the time domain adopting for example the Crank-Nicolson integration scheme. Details on the numerical implementation are available in [34]. The homogenized matrices in Eqs. (22) and (23) follow directly from the meso-scale solution for a given macroscopic time increment. Because of a strong nonlinearity arising on both scales the two problems (macro-meso) must be solved iteratively by the Newton-Raphson method, see $[21,22,23]$ for further reference.

\subsection{Numerical Examples}

A fully coupled multi-scale analysis of a twodimensional segment of Charles Bridge in Prague has been carried out in [23] with emphases on parallel computing. This study will be extended herein by quantifying the influence of the boundary, loading and initial conditions when moving down from the macro-scale to the meso-scale.

\subsubsection{Boundary Conditions}

To begin, recall Eq. (20)

$\left\langle\boldsymbol{\nabla} \theta^{*}(\boldsymbol{x})\right\rangle_{\square}=\frac{1}{\left|\Omega_{\square}\right|} \int_{\Gamma_{\square}} \theta^{*}(\boldsymbol{x}) \boldsymbol{v}(\boldsymbol{x}) \mathrm{d} \Gamma(\boldsymbol{x})=0$, 
being satisfied providing either the fluctuation part of the temperature field equals zero or the periodic boundary conditions, i.e. the same values of $\theta^{*}$ on opposite sides of a rectangular PUC, are enforced on $\Gamma_{\square}$.

Such conditions are easy to impose if searching the solution in terms of the fluctuation part of the temperature or moisture fields. The macroscopic constant gradients $\nabla \Theta$ and $\boldsymbol{\nabla} \Phi$, see Fig. (7a), are then directly used to load the unit cell [10]. However, this is mostly not possible with the application of commercial codes. In such a case, the constant gradients are introduced by enforcing a linear variation of the homogeneous part of local fields $\Theta^{\mathrm{hom}}(\boldsymbol{x}), \Phi^{\mathrm{hom}}(\boldsymbol{x})$. This is achieved by prescribing directly the Dirichlet boundary conditions along all edges of PUC, i.e. $\theta(x)=\Theta^{\text {hom }}(x), \varphi(x)=$ $\Phi^{\text {hom }}(\boldsymbol{x})$ (see Fig. (7b)) are specified on the boundary $\Gamma_{\square}$ of PUC. The periodic boundary conditions are then prescribed with the help of multi-point constraints.

In doing so, observe in Fig. (6) that for a pair of points (e.g. $A$ - master and $a$ - slave) located on the opposite sides of PUC the following relations hold:

$\theta_{A}=\left(\frac{\partial \Theta}{\partial y}\right) y_{A}+\theta_{A}^{*}+\Theta\left(\boldsymbol{X}_{0}\right), \theta_{a}=\left(\frac{\partial \Theta}{\partial x}\right) L+\left(\frac{\partial \Theta}{\partial y}\right) y_{a}+\theta_{a}^{*}+$ $\Theta\left(\boldsymbol{X}_{0}\right)$.

Taking into account the fact that the fluctuation field $\theta^{*}$ satisfies the periodicity condition

$\theta_{a}^{*}=\theta_{A}^{*}$,

and subtracting corresponding terms on the opposite edges, we finally obtain (compare with [21]

$\left(\frac{\partial \Theta}{\partial x}\right) L=\theta_{a}-\theta_{A}=\theta_{2}-\theta_{1}$,
$\left(\frac{\partial \Theta}{\partial y}\right) H=\theta_{b}-\theta_{B}=\theta_{3}-\theta_{1}$,

where $\theta_{1}, \theta_{2}$ and $\theta_{3}$ are the temperatures at the control points 1,2 and 3 seen in Fig. (6). The same conditions apply to the relative humidity $\varphi$ as well.

\subsubsection{Loading and Initial Conditions}

An illustrative example presented here considers a finite element mesh consisting of 108 macro-elements each representing a single meso-problem with assigned periodic boundary conditions. Its geometry together with the specific

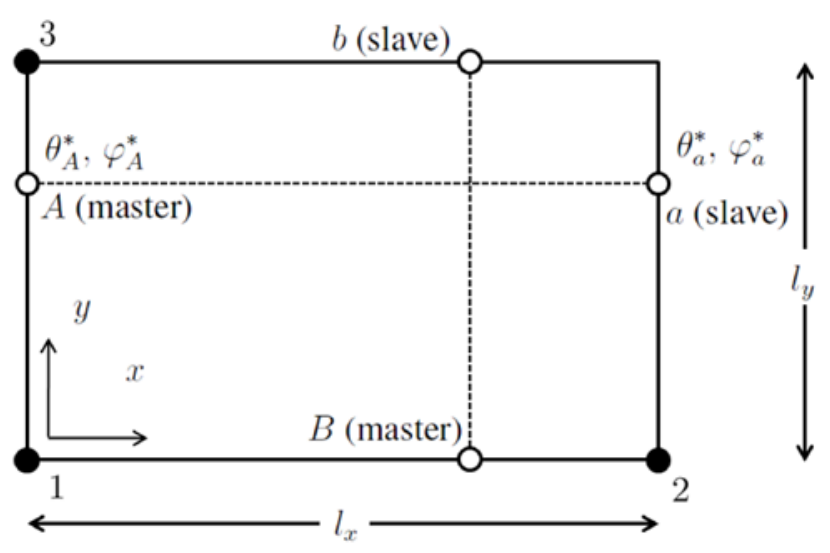

Fig. (6). Periodic boundary conditions.

macroscopic loading conditions are shown in Fig. (5).

The initial temperature $\theta_{\text {in }}=14\left[{ }^{\circ} \mathrm{C}\right]$ and the moisture $\varphi_{\text {in }}$ $=0.5[-]$ were assigned to the whole domain. The following boundary and loading conditions were imposed: the left boundary of the domain was submitted to exterior loading conditions $\theta_{\text {ext }}=5\left[{ }^{\circ} \mathrm{C}\right]$ and $\varphi_{\text {ext }}=0.5$ [-], while the opposite side was submitted to interior loading conditions $\theta_{\text {int }}=24$ $\left[{ }^{\circ} \mathrm{C}\right]$ and $\varphi_{\text {int }}=0.8[-]$. Zero flux boundary conditions were assumed for horizontal edges.

The material parameters in Table $\mathbf{2}$ were obtained from a set of experimental measurements providing the hygric and thermal properties of mortars and bricks/stones, which have been used in the reconstruction works of historical buildings in the Czech Republic, see [10, 35].

With reference to Section 3.2.1 we consider two types of loading conditions. Fig. (7a) assumes the loading in terms of macroscopic temperature and moisture gradients to provide local fluctuations upon submitting decompositions (17) and (18) into Hill's averaging condition (consider for simplicity a steady-state heat conduction problem only)

$\left\langle\delta \boldsymbol{\nabla} \theta^{\mathrm{T}} \boldsymbol{q}\right\rangle=0$, to get

$\left\langle\delta \nabla \theta^{* \mathrm{~T}} \lambda \boldsymbol{\nabla} \theta^{*}\right\rangle=-\left\langle\delta \boldsymbol{\nabla} \theta^{* \mathrm{~T}} \lambda \boldsymbol{\nabla} \Theta\right\rangle$.

The periodic boundary conditions are then easily introduced by prescribing the same code numbers, in the FEM

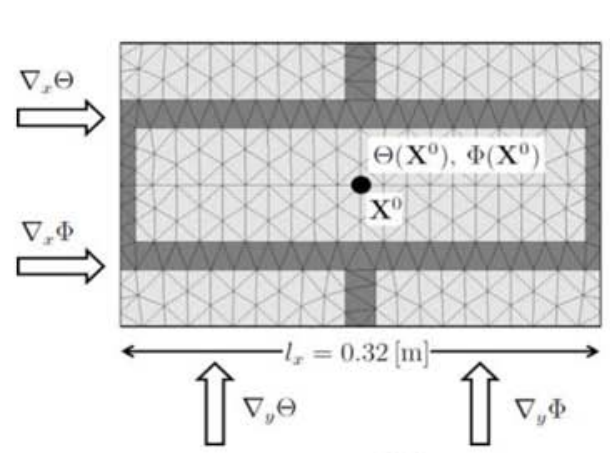

(a)

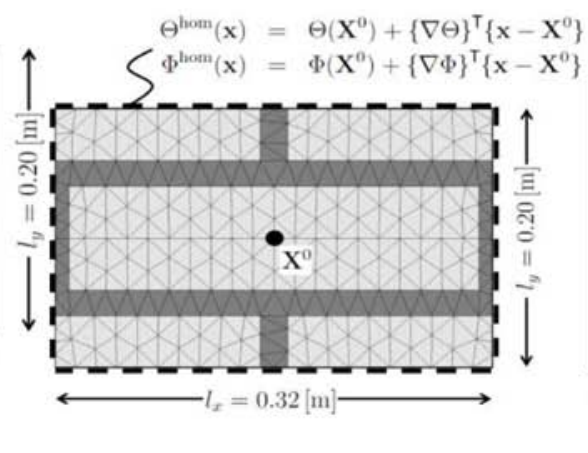

(b)

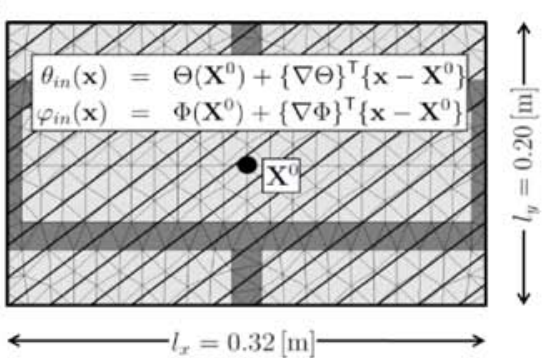

(c)

Fig. (7). Different loading schemes of PUC on meso-scale: (a) prescribed macroscopic gradients (loading conditions I, labeled as NST ${ }^{1}$ ), (b) prescribed macroscopic temperatures and relative humidities (loading conditions II, labeled as NST ${ }^{2}$ ); (c) Assumed initial conditions. 
language, to the homologous nodes on the opposite faces of the rectangular unit cell. Fig. (7b) then presents fully prescribed Dirichlet boundary conditions applicable with commercial codes when searching the solution directly in terms of the local fields $\theta, \varphi$. The periodic boundary conditions are then enforced indirectly using Eq. (27).

Apart from loading conditions, the two types of initial conditions seen in Fig. (7c) are also examined. In particular, either a linear variation of the homogeneous part of fields $\theta_{\text {in }}(\boldsymbol{x})=\Theta^{\mathrm{hom}}(\boldsymbol{x}), \varphi_{\text {in }}(\mathrm{x})=\Phi^{\mathrm{hom}}(\boldsymbol{x})$ is assumed or the macroscopic temperature $\theta_{\text {in }}(\boldsymbol{x})=\Theta\left(\boldsymbol{X}^{0}\right)$ and moisture $\varphi_{\text {in }}(\boldsymbol{x})=$ $\Phi\left(\boldsymbol{X}^{0}\right)$ calculated at the reference point $\boldsymbol{X}^{0}$ at the end of the current macroscopic time step are assigned to the whole PUC. Note that the former one arise naturally from Eqs. (17) and (18) when setting the fluctuation fields equal to zero at time $t=0$.

\section{Meso-scale Analysis}

In the first example attention is dedicated to the solution of a coupled transient heat and moisture problem solely on the meso-scale. First, the regular periodic unit cell, PUC in Fig. (5), was loaded by the highest temperature and moisture gradients obtained in the course of actual multi-scale analysis discussed later in this section. The following numerical values were considered; initial conditions $\left(\Theta\left(\boldsymbol{X}^{0}\right)=17\left[{ }^{\circ} \mathrm{C}\right]\right.$, $\left.\Phi\left(\boldsymbol{X}^{0}\right)=0.15,0.55,0.85[-]\right)$ and boundary conditions $(\{\boldsymbol{\nabla} \Theta$ $=\left\{\nabla_{\mathrm{x}} \Theta, \nabla_{\mathrm{y}} \Theta\right\}=\{5.0,1.0\}\left[{ }^{\circ} \mathrm{Cm}^{-1}\right], \nabla \Phi=\left\{\nabla_{\mathrm{x}} \Phi, \nabla_{\mathrm{y}} \Phi\right\}=$ $\left.\{0.1,0.05\}\left[\mathrm{m}^{-1}\right]\right)$.

Fig. (8) shows evolution of local temperature and moisture fields along the horizontal centerline of PUC as a function of time derived from two types of loading conditions. Periodic boundary conditions and linearly varying initial conditions with $\Phi\left(\boldsymbol{X}^{0}\right)=0.55$ [-] were considered. It is shown that for the adopted extreme gradients the steady state solution, plotted as solid lines, is reached in about 15 hours irrespective of loading conditions.

Further to this subject, we also suggest invariance of the solution to the assumed initial conditions as depicted in Fig. (9). This becomes evident once realizing a nonlinearity of Eqs. (12) and (14) taken into account through the application of Newton-Raphson iteration scheme. Clearly, the initial difference in the solution error attributed to the difference in initial conditions is wiped out already in the first load (time) increment upon arriving at equilibrium.

The influence of the degree of material nonlinearity of the present constitutive model is partially seen in Fig. (11) showing evolution of the selected effective material properties as a function of time for three values of initial macroscopic moisture $\Phi\left(\boldsymbol{X}^{0}\right)$.

Functional dependence of some material parameters on moisture is plotted in Fig. (10) for illustration. The complete set is available, e.g. in [34, 36]. It is evident that for effective properties the time to reach the steady state solution is considerably shorter than for local temperatures and humidities, recall Fig. (8). It is even more important to realize that the difference between effective steady state parameters and effective parameters within a transient regime is essentially negligible. This can be attributed to a relatively small difference between local fields pertinent to steady state and various stages of transient solutions as evident in Fig. (8). In other words, the material nonlinearity observed in Fig. (10) does not play in this case a significant role, thus promoting the steady state solution from the macroscopic point of view as sufficiently accurate.

Finally, to address the influence of the degree of material heterogeneity we repeated the same study employing the irregular periodic unit cell (SEPUC in Fig. 5). Figs (12a,b) show evolution of relative humidity along the SEPUC centerline at various times clearly identifying the material boundaries. While the evolution trend is similar to the results presented in Fig. (8) for PUC, the time to attain steady state solution slightly increases. Evolutions of the selected effective moisture terms is plotted in Figs $(\mathbf{1 2 c}, \mathbf{d})$ confirming again a negligible difference between steady state and transient analyses. While the degree of heterogeneity is pronounced only slightly, the influence of initial conditions on the prediction of effective properties is significant.

\section{Multi-scale Analysis}

Combining all the previous results suggests that for a reasonably small macroscopic time increment (from one to two hours sufficient to reach the steady state conditions on the meso-scale) the influence of non-local terms in Eqs. (22) and (23) should be negligible. Therefore, the macroscopic response should be invariant with respect to the adopted

Table 2. Material Parameters of Individual Phases

\begin{tabular}{|c|l|l|r|r|}
\hline Parameter & & & Brick & Mortar \\
\hline \hline$w_{f}$ & {$\left[\mathrm{kgm}^{-3}\right]$} & free water saturation & 229.30 & 160.00 \\
\hline$w_{80}$ & {$\left[\mathrm{kgm}^{-3}\right]$} & water content at $\varphi=0.8[-]$ & 141.68 & 22.72 \\
\hline$\lambda_{0}$ & {$\left[\mathrm{Wm}^{-1} \mathrm{~K}^{-1}\right]$} & thermal conductivity & 0.25 & 0.45 \\
\hline$b_{t c s}$ & {$[-]$} & thermal conductivity supplement & 9 \\
\hline$\rho_{s}$ & {$\left[\mathrm{kgm}^{-3}\right]$} & bulk density & 10 & 1690 \\
\hline$\mu$ & {$[-]$} & water vapor diffusion resistance & 1670 \\
\hline$A$ & {$\left[\mathrm{kgm}^{-2} \mathrm{~s}^{-0.5}\right]$} & water absorption coefficient & 9.63 \\
\hline$c_{s}$ & {$\left[\mathrm{Jkg}^{-1} \mathrm{~K}^{-1}\right]$} & specific heat capacity & 0.51 \\
\hline
\end{tabular}


analysis carried out on meso-scale whether the transient or steady state. This is evident from the results plotted in Fig (13) showing the evolution of macroscopic temperatures and relative humidities at selected nodes of mesh, see Fig. (5).

This result is quite encouraging particularly with reference to the analysis of large historical structures, since avoiding a transient analysis on each mesoscopic unit cell may considerably reduce the computational cost.

\section{CONCLUSIONS}

The present paper gives a brief overview of two particular aspects of the modeling of historical masonry structures,

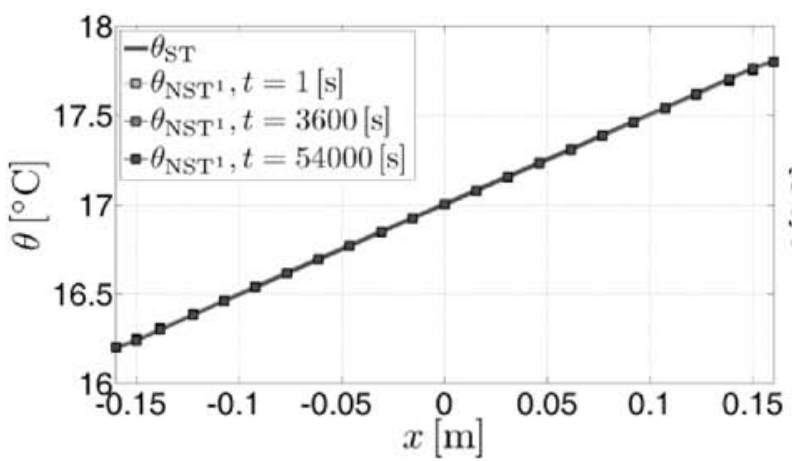

(a)

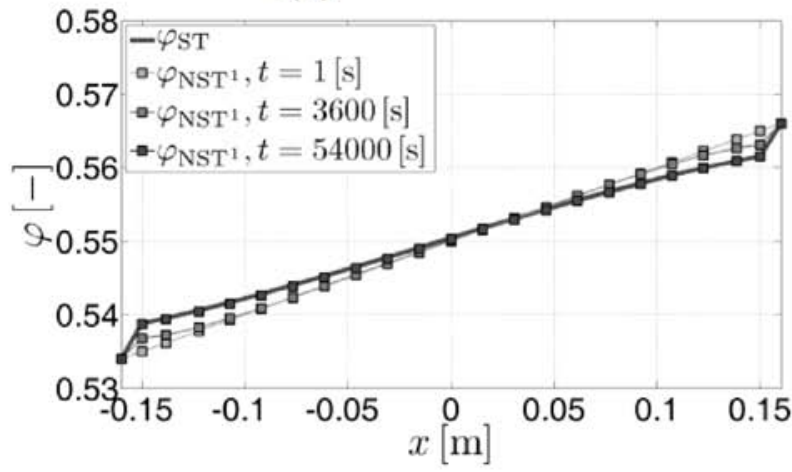

(c) which show a certain degree of irregularity on the mesoscale. This observation promotes application of so called Statistically Equivalent Periodic Unit Cell being sufficiently simple in comparison to, yet sufficiently representative of, a real masonry. This issue was addressed first in Section 2 to see that under steady state and linear conditions the degree of heterogeneity, associated with a given meso-structure, may not play a significant role in the prediction of effective macroscopic response, recall Table $\mathbf{1}$. On the contrary, the morphological details will become important once considering a nonlinear response crucially dependent on the actual distribution of local fields.

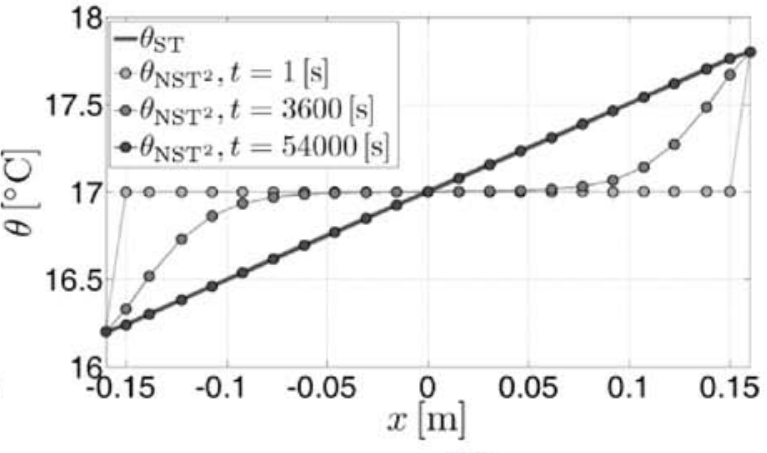

(b)

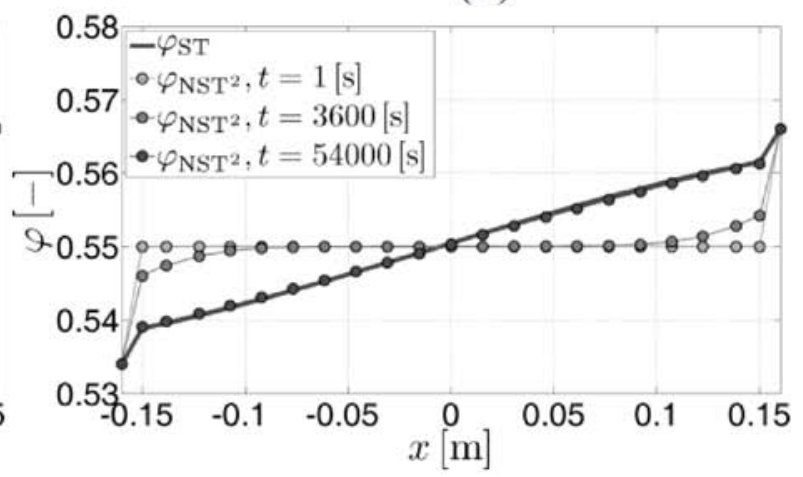

(d)

Fig. (8). Evolution of local fields (PUC): (a,b) temperature, (c,d) moisture; (a,c) Loading conditions I (Fig. 7(a)), (b,d) Loading conditions II (Fig. 7(b)).

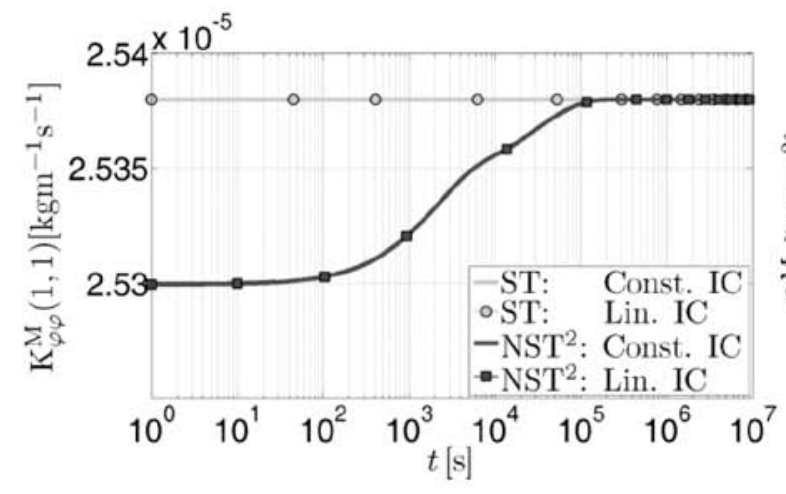

(a)

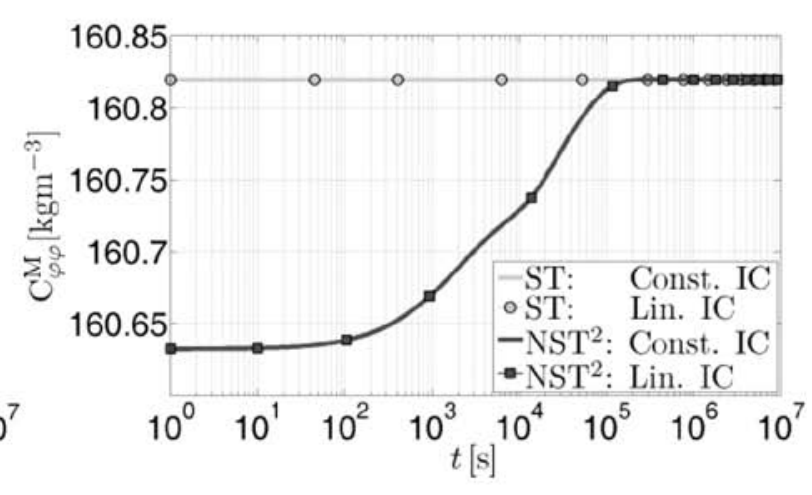

(b)

Fig. (9). Influence of initial conditions (constant or linearly varying homogeneous part of local fields); Evolution of effective moisture terms as a function of time: (a) effective conductivity term, (b) effective storage term. 


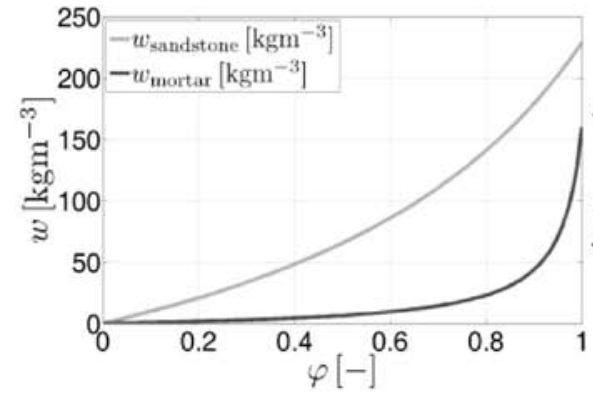

(a)

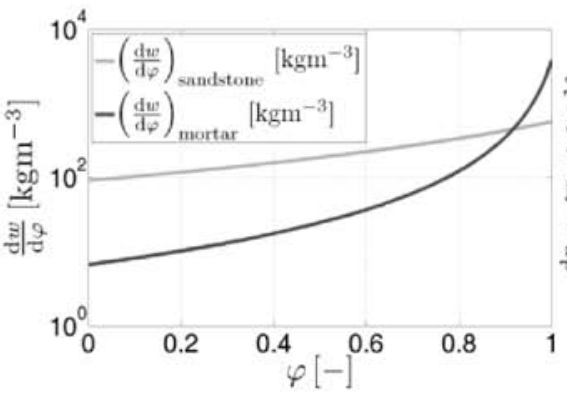

(b)

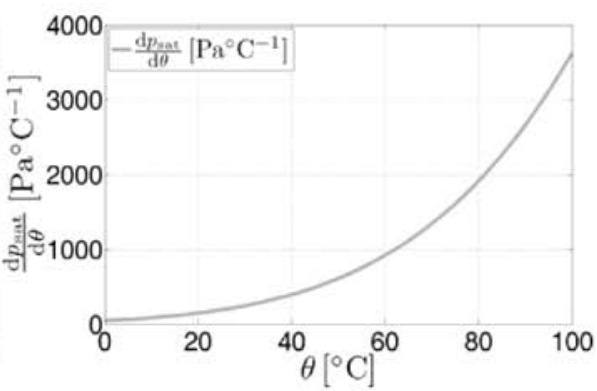

(c)

Fig. (10). (a) Variation of water content as a function of relative humidity, (b) Variation of phase moisture storage function as a function of relative humidity, (c) Variation of slope of saturation pressure as a function of temperature.

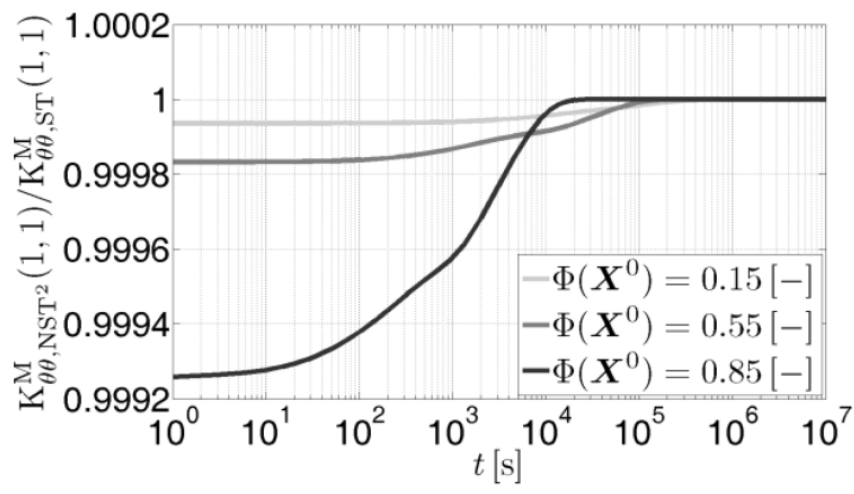

(a)

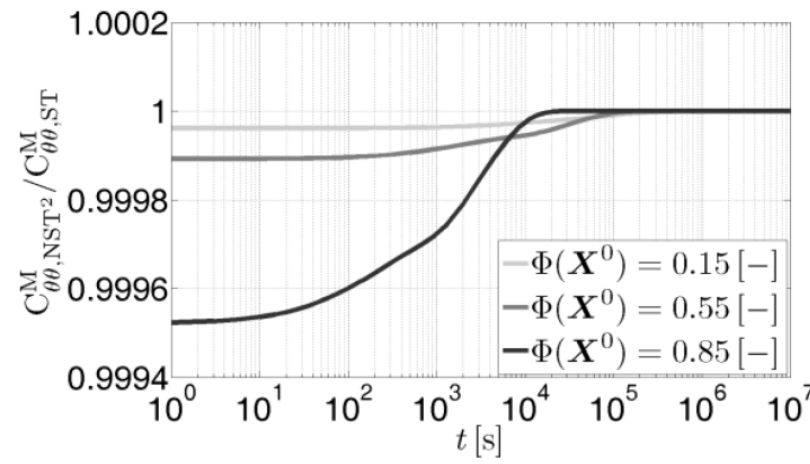

(c)

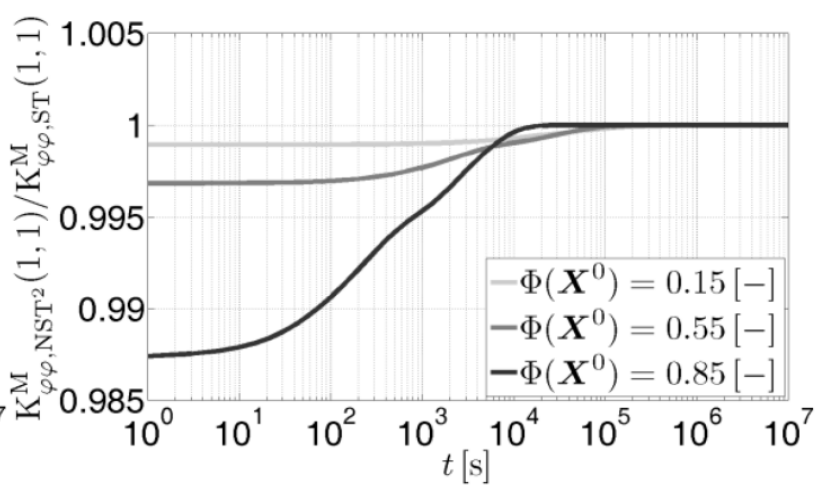

(b)

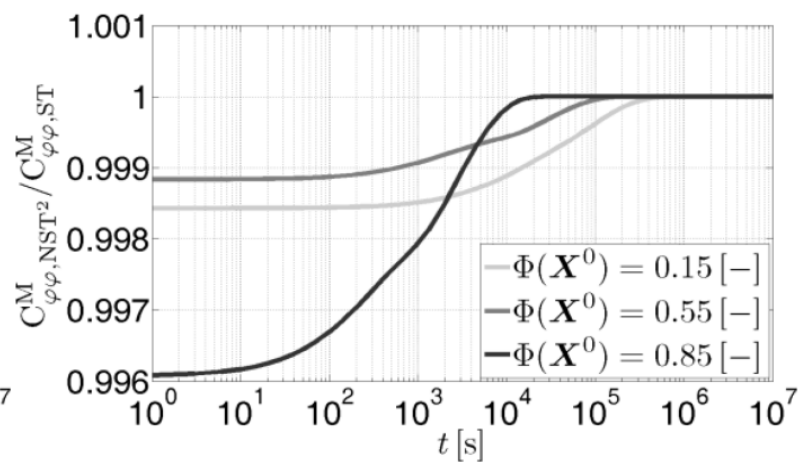

(d)

Fig. (11). Evolution of homogenized material parameters as function of time (PUC): (a) $K_{\theta \theta,(1,1)}^{\mathrm{M}}$, (b) $K_{\varphi \varphi,(1,1)}^{\mathrm{M}}$, (c) $C_{\theta \theta}^{\mathrm{M}}$, (d) $C_{\varphi \varphi}^{\mathrm{M}}$

The latter comment was partially examined next in Section 3 devoted to the nonlinear fully coupled multi-scale analysis of simultaneous heat and moisture transport. The principal result of this study is seen in the possibility of deriving the instantaneous, time dependent, macroscopic response from a steady-state analysis performed on the lower scale reflecting all morphological details. However, keep in mind that this finding is strictly valid for the present problem being a collection of the selected climatic conditions, material composition and the assumed constitutive model, and should not be generalized. For other cases the theoretically predicted dependence of macroscopic response on the actual RVE size may prove non-negligible [22, 23].

It is our present interest to exploit the two advancement in computational efficiency (SEPUC, steady state meso-scale problem) in the analysis of full scale 3D model of Charles Bridge. Special attention will be devoted to the implementation efficiency in the framework of hybrid parallel computing.

\section{CONFLICTS OF INTEREST}

The authors confirm that this article content has no conflicts of interest.

\section{ACKNOWLEDGEMENT}

The financial support of the GACR grants P105/11/0411 (J.S. and J.Z.) and P105/11/0224 (M.Š.) is gratefully acknowledged. In addition, work by J.Z. was partially supported by the European Regional Development Fund in the 


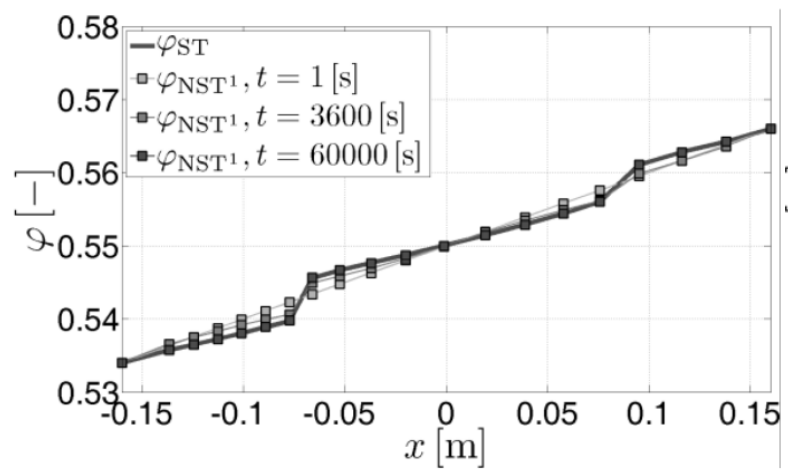

(a)

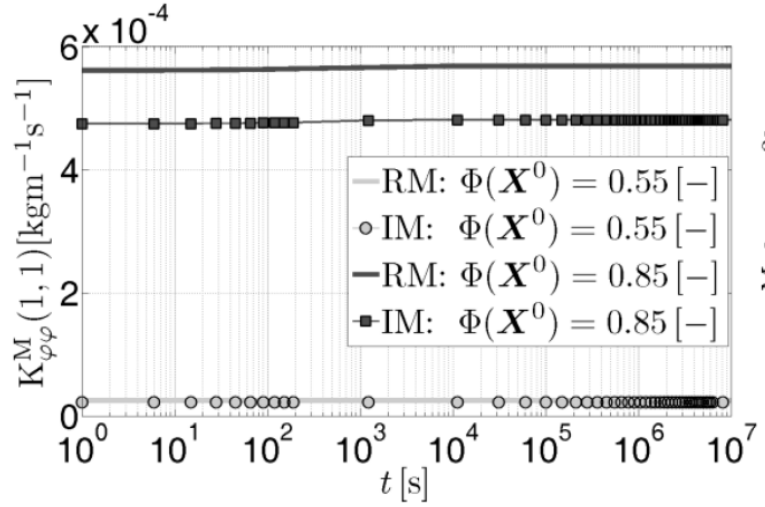

(c)

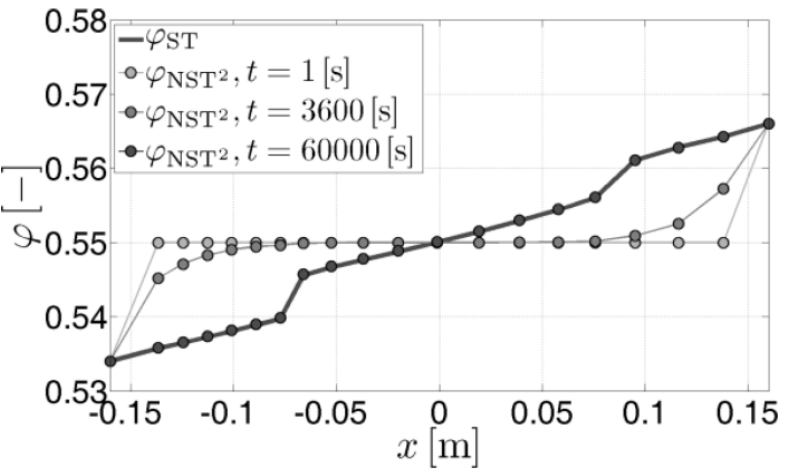

(b)

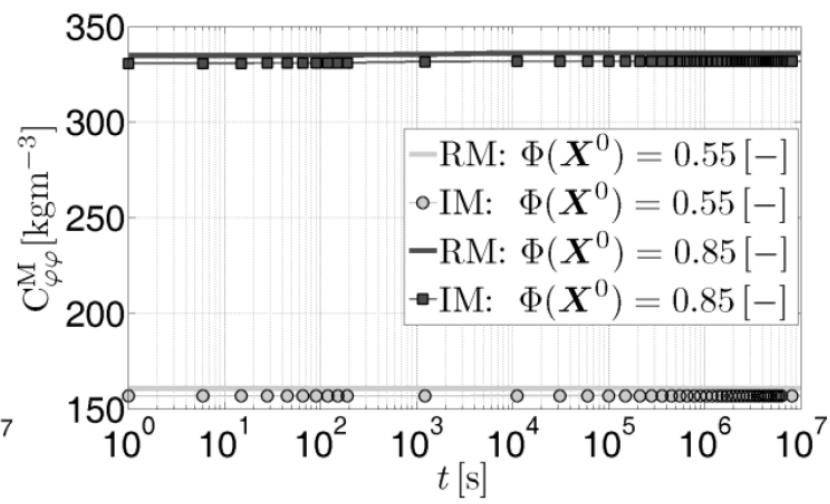

(d)

Fig. (12). Evolution of local moisture (SEPUC): (a) loading conditions I, (b) loading conditions II; Evolution of homogenized material parameters as s function of time: (c) $K_{\varphi \varphi,(1,1)}^{\mathrm{M}},(\mathbf{d}) C_{\varphi \varphi}^{\mathrm{M}}$.

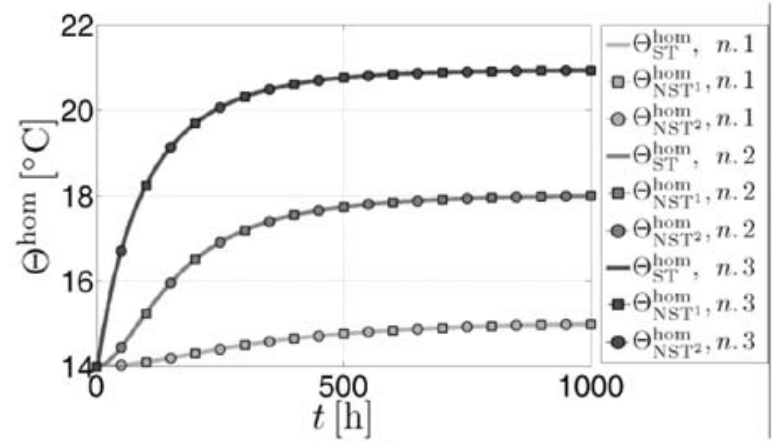

(a)

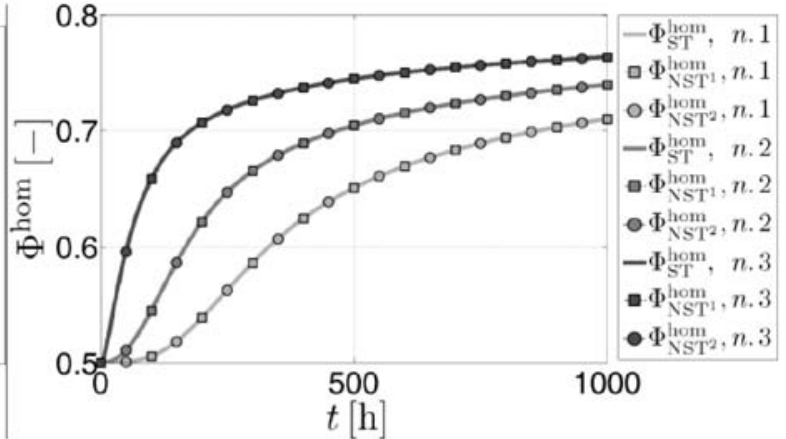

(b)

Fig. (13). Comparis on of different macrostructural computations at selected nodes - (a) temperature evolution, (b) moisture evolution.

IT4Innovations Centre of Excellence project (CZ.1.05/1.1. 00/02.0070). We also thank Jaroslav Vondřejc (CTU in Prague) for his assistance with the FFT-based simulations.

\section{REFERENCES}

[1] P. B. Lourenço, "Computations on historic masonry structures", Prog. Struct. Eng. Mater., vol. 4, no 3, pp. 301-319, 2002.

[2] G. I. B. Rankin, A. Thompson, and R. D. N. Robinson, "Temperature effects on copper-clad brickwork dome roof", in Proceedings of ICE - Struct. Buildings, vol. 158, no. 6, pp. 343-353, 2005.

[3] J. Witzany, T. Čejka, and R. Zigler, "Failure resistance of the historic stone bridge structure of Charles Bridge. I: Susceptibility to nonstress effects”, J. Perform. Construct. Facil., vol. 22, no. 2, pp. 71-82, 2008.
[4] P. Fajman, J. Máca, and P. Beran, "Influence of temperature chan ges on the Vladislava Hall vault”, Acta Geodynamica et Geomaterialia, vol. 7, no. 2, pp. 219-225, 2010.

[5] A. Anthoine, "Derivation of the in-plane elastic characteristics of masonry through homogenization theory”, Int. J. Solids Struct., vol. 34, no. 11, pp. 137-163, 1995.

[6] P. B. Lourenço, G. Milani, A. Tralli, and A. Zucchini, “Analysis of masonry structures: review of and recent trends in homogenization techniques”, Can. J. Civ. Eng., vol. 34 no. 11, pp. 1443-1457, 2007.

[7] T. J. Massart, R. H. J. Peerlings, and M. G. D. Geers, “An enhanced multi-scale approach for masonry wall computations with localization of damage”, Int. J. Numer. Meth. Eng., vol. 69 no. 5, pp. 1022-1059, 2007.

[8] J. Šejnoha, M. Šejnoha, J. Zeman, J. Sýkora, and J. Vorel, “A mesoscopic study on historic masonry”, Struct. Eng. Mech., vol. 30, no. 1, pp. 99-117, 2008. 
[9] J. Sýkora, J. Vorel, T. Krejčí, M. Šejnoha, and J. Šejnoha, “Analysis of coupled heat, and moisture transfer in masonry structures", Mater. Struct., vol. 42, no. 8, pp. 1153-1167, 2008.

[10] J. Sýkora, M. Šejnoha, and J. Šejnoha, "Homogenization of coupled heat and moisture transport in masonry structures including interfaces”, App. Math. Comput., 2011. (In Press).

[11] J. Zeman, J. Novák, M. Šejnoha, and J. Šejnoha, "Pragmatic multiscale and multiphysics analysis of Charles Bridge in Prague” Eng. Struct., vol. 30, no. 11, pp. 3365-3376, 2008.

[12] R. Hill, "Elastic properties of reinforced solids - Some theoretical principles”, J. Mech. Phys. Solids, vol. 11, pp. 357-372, 1963.

[13] G. L. Povirk, "Incorporation of microstructural information into models of two-phase materials", Acta Metallurgica et Materialia, vol. 43, no. 8, pp. 3199-3206, 1995.

[14] C. L. Y. Yeong and S. Torquato, "Reconstructing random media", Phys. Rev. E, vol. 57, no. 1, pp. 495-506, 1998.

[15] J. Zeman and M. Šejnoha, "Numerical evaluation of effective properties of graphite fiber tow impregnated by polymer matrix", $J$. Mech. Phys. Solids, vol. 49, no. 1, pp. 69-90, 2001.

[16] M. Šejnoha and J. Zeman, "Overall viscoelastic response of random fibrous composites with statistically quasi uniform distribution of reinforcements", Comp. Methods Appl. Mech. Eng., vol. 191, no. 44, pp. 5027-5044, 2002.

[17] J. Zeman and M. Šejnoha, "Homogenization of balanced plain weave composites with imperfect microstructure. Part I. Theoretical formulation”, Int. J. Solids Struct., vol. 41, no. 21-22, pp. 65496571, 2004.

[18] J. Vorel, J. Zeman, and M. Šejnoha, "Homogenization of plain weave composites with imperfect microstructure: Part II-Analysis of real-world materials”, Int. J. Multiscale Comput. Eng., 2012. (In Press).

[19] H. Lee, M. Brandyberry, A. Tudor, and K. Matouš, "Threedimensional reconstruction of statistically optimal unit cells of polydisperse particulate composites from microtomography", Phys. Rev. E Stat, Nonlin. Soft Matter Phys., vol. 80, no. 6, pp. 061301-1061301-12, 2009.

[20] J. Schröder, D. Balzani, and D. Brands, “Approximation of random microstructures by periodic statistically similar representative volume elements based on lineal-path functions", Arch. Appl. Mech., vol. 81, no. 7, pp. 975-997, 2011.

[21] I. Özdemir, W. A. M. Brekelmans, and M. G. D. Geers, "Computational homogenizaion for heat conduction in heterogeneous solids", Int. J. Numer. Methods Eng., vol. 2, pp. 185-204, 2008.

[22] F. Larsson, K. Runesson, and F. Su, "Variationally consistent computational homogenization of transient heat flow", Int. J. Numer. Methods Eng., vol. 81, pp. 1659-1686, 2010.

[23] J. Sýkora, T. Krejčí, J. Kruis, and M. Šejnoha, "Computational homogenization of, non-stationary transport processes in masonry structures”, J. Comput. Appl. Math., vol. 236, no. 18, pp. 47454755, 2012.

[24] S. Torquato, Random heterogeneous materials: Microstructure and macroscopic properties. Springer-Verlag: USA, 2002.

[25] J. Gajdošík J. Zeman, and M. Šejnoha, "Qualitative analysis of fiber composite microstructure: Irfluence of boundary conditions", Probab. Eng. Mech., vol. 21, no. 4, pp. 317-329, 2006.

[26] O. Hrstka, A. Kučerová, M. Lepš, and J. Zeman, "A competitive comparison of different types of evolutionary algorithms”, Comput. Struct., vol. 81, no. 18-19, pp. 1979-1990, 2003.

[27] K. Matouš, M. Lepš, J. Zeman, and M. Šejnoha, “Applying genetic algorithms to selected topics commonly encountered in engineering practice”, Comput. Methods Appl. Mech. Eng., vol. 190, no. 13-14, pp. 1629-1650, 2000.

[28] G. W. Milton, The Theory of Composites, volume 6 of Cambridge Monographs on Applied and Computational Mathematics. Cambridge University Press: Cambridge, 2002.

[29] H. Moulinec and P. Suquet, "A fast numerical method for computing the linear and nonlinear mechanical properties of composites", Comptes rendus de l'Académie des sciences. Série II, Mécanique, physique, chimie, astronomie, vol. 318, no. 11, pp. 1417-1423, 1994.

[30] J. Zeman, J. Vondřejc, J. Novák, and I. Marek, "Accelerating a FFT-based solver for numerical homogenization of periodic media by conjugate gradients", J. Comput. Phys., vol. 229, no. 21, pp. 8065-8071, 2010.

[31] J. Saranen and G. Vainikko, Periodic Integral and Pseudodifferential Equations with Numerical Approximation. Springer Monographs in Mathematics, Springer-Verlag: USA, 2002.

[32] H. M. Künzel, "Simultaneous Heat and Moisture Transport in Building Components”, Technical report, Fraunhofer IRB Verlag Stuttgart, 1995.

[33] H. M. Künzel and K. Kiessl, "Calculation of heat and moisture transfer in exposed building components”, Int. J. Heat Mass Trans., vol. 40, pp. 159-167, 1997.

[34] J. Sýkora, "Multiscale modeling of transport processes in masonry structures", PhD thesis, Czech Technical University in Prague, 2010.

[35] M. Pavlíková, Z. Pavlík and R. Černý, "Hygric and thermal properties of materials of historical masonry," in Proceedings of the 8th Symposium on Building Physics in the Nordic Countries, 2008.

[36] M. Šejnoha and J. Sýkora, "Hierarchical modeling of transport processes in heterogeneous materials: Application to historical masonry structures”, in Selected Chapters of Mechanics of Composite Materials II, K. Kotrasová and E. Kormaníková, Ed., Czech Technical University: Prague, 2012, pp. 1-48. 